\title{
Wirkt DMP qualitätsverbessernd? - Eine empirische Untersuchung mittels Routinedaten
}

\section{Hintergrund \\ $\nabla$}

Seit Einführung der Disease-Management-Programme (DMP) im Jahr 2003 wurden für die mit Abstand umfangreichste Bündelintervention in Deutschland mehrere Milliarden Euro aufgewendet, allein im Jahr 2009 waren es ca. 1,1 Milliarden Euro. Bis heute fehlt eine fundierte wissenschaftliche Evaluation. Nachdem längere Zeit ein Nutzen vermutet wurde, mehren sich in letzter Zeit Zweifel an der Sinnhaftigkeit der DMP in ihrer heutigen dokumentationslastigen Form. Neben den unlängst publizierten ernüchternden Studienergebnissen von Prof. Berthold [1], den Ausführungen von Frau Prof. Landenberger hinsichtlich fehlender Belege für medizinische Vorteile, den Zweifeln an einer Kosteneinsparung von Prof. Busse [2] und der radikalen Forderung von Prof. Wasem nach einem Förderungsstopp für DMP haben sich - neben der Techniker Krankenkasse (TK) - auch mehrere große Krankenkassen der Thematik angenommen und konnten dabei entweder einen Programmeffekt nicht nachweisen oder sehen den Nutzen eher für eine Subpopulation. Angesichts vielfältiger Selektionseffekte hat das WINEG mit innovativer Methodik untersucht, inwieweit das DMP „Diabetes mellitus Typ 2“ („T2DM“) das Outcome für TK-Versicherte verbessert oder hilft, Kosten einzusparen (ausführliche Studie siehe [3]).

\section{Methoden}

\section{$\nabla$}

Untersucht wurden Routinedaten der Techniker Krankenkasse aus den Jahren 2006 bis 2008 mit harten Endpunkten wie z.B. der Inzidenz von Schlaganfall, Herzinfarkt oder Niereninsuffizienz. Bewusst verzichtet wurde auf eine Auswertung der qualitativ diskussionswürdigen Daten aus der DMP-Dokumentation. Ausgehend von einem „Propensity Score Interval Matching“ wurde ein ausgefeiltes Kontrollgruppendesign entwickelt und angewendet. Die dabei berücksichtigten Variablen waren Alter, Geschlecht, Ausbildung, Berufstätigkeit, Pflegestufe, Komorbiditäten, „Defined Daily Dose“ (DDD) sowie Pharma- und Krankenhauskosten im Jahr vor der (möglichen) Einschreibung der Versicherten in das DMP.

\section{Ergebnisse

$$
\nabla
$$

Hinsichtlich der Inzidenz von Komorbiditäten, Fußamputationen, Inanspruchnahme von Pharmaka, Krankenhauskosten und Notfalleinweisungen zeigten sich mit Ausnahme häufigerer
Medikamentenverordnungen in der Gruppe der DMP-Teilnehmer keine deutlichen Unterschiede zu Teilnehmern der Kontrollgruppe. Die Zahl der Notfalleinweisungen und die Kosten für stationäre Aufenthalte waren bei den DMP-Teilnehmern etwas niedriger. Die DMP-Teilnehmer haben in allen untersuchten Quartalen mehr Arzneimittelverordnungen eingelöst, häufiger niedergelassene Ärzte kontaktiert und mehr EBMLeistungen in Anspruch genommen. Kosteneinsparungen bei den DMP-Teilnehmern waren nicht erkennbar.

\section{Diskussion $\nabla$}

Der Nutzen des vom Volumen her wichtigsten DMP „T2DM“ ist momentan in Deutschland nicht eindeutig belegt. Auch die WINEG-Studie lässt einen deutlichen Nutzen des DMP nicht erkennen. Angesichts der noch immer ungeklärten Studienlage und der immensen Programmkosten fordert die TK die bestmögliche Evidenz zur Nutzenbewertung von DMP. Diese ist durch eine kassenübergreifende Evaluation von Routinedaten der Gesetzlichen Krankenversicherungen (GKV) vorstellbar, erfolgt idealerweise jedoch durch eine prospektive randomisierte Studie. Sollte nach gründlicher Prüfung ein akzeptables Kosten-Nutzen-Verhältnis nicht darstellbar sein, müssen die Bestandteile der Bündelintervention DMP einzeln geprüft, die Einschreibepraxis angepasst (vom Gießkannen- zum Pareto-Prinzip), in jedem Fall jedoch Fehlallokationen zugunsten besser geeigneter Interventionen abgestellt werden.

Autorenerklärung: Das Wissenschaftliche Institut der TK für Nutzen und Effizienz im Gesundheitswesen (WINEG) hat zur Aufgabe, die Wertigkeit von Innovationen und neuen programmatischen Ansätzen innerhalb der GKV zu hinterfragen. Die Autoren erklären, dass aufgrund ihrer Zugehörigkeit zur Techniker Krankenkasse ein potenzieller Interessenskonflikt im Sinne der Richtlinien des International Committee of Medical Journal Editors besteht.

\section{Literatur}

1 Berthold HK, Bestehorn KP, Jannowitz C, Krone W, Gouni-Berthold I. Disease Management Programs in Type 2 Diabetes: Quality of Care. Am J Manag Care 2011; 17: 393-403

2 Busse R. Bekämpfung chronischer Krankheiten und Versorgung chronisch Kranker - international. Die BKK 2011; 3: 142-145

3 Linder R, Ahrens S, Köppel D, Heilmann T, Verheyen F. Nutzen und Effizienz des Disease-ManagementProgramms Diabetes Mellitus Typ 2. Dtsch Ärztebl 2011; 108: 155-162
R. Linder

Qualitätsmanagement

Schlüsselwörter

Disease-ManagementProgramme

Diabetes mellitus Typ 2 Kontrollgruppendesign

Propensity Score Interval Matching

Keywords

disease management programs

Type 2 diabetes mellitus

control group design

propensity score interval matching

Institut

Wissenschaftliches Institut der TK für Nutzen und Effizienz im Gesundheitswesen (WINEG), Hamburg

Bibliografie

DOI 10.1055/s-0031-1286094

Dtsch Med Wochenschr 2011; 136: S65 - (c) Georg Thieme Verlag KG Stuttgart - New York . ISSN 0012-0472

Korrespondenz Prof. Dr. Roland Linder WINEG | Wissenschaftliches Institut der TK für Nutzen und Effizienz im Gesundheitswesen Bramfelder Straße 140 22305 Hamburg

Tel. 040/6909-2338

Fax 040/6909-2307 eMail prof.dr.roland.linder@ wineg.de 